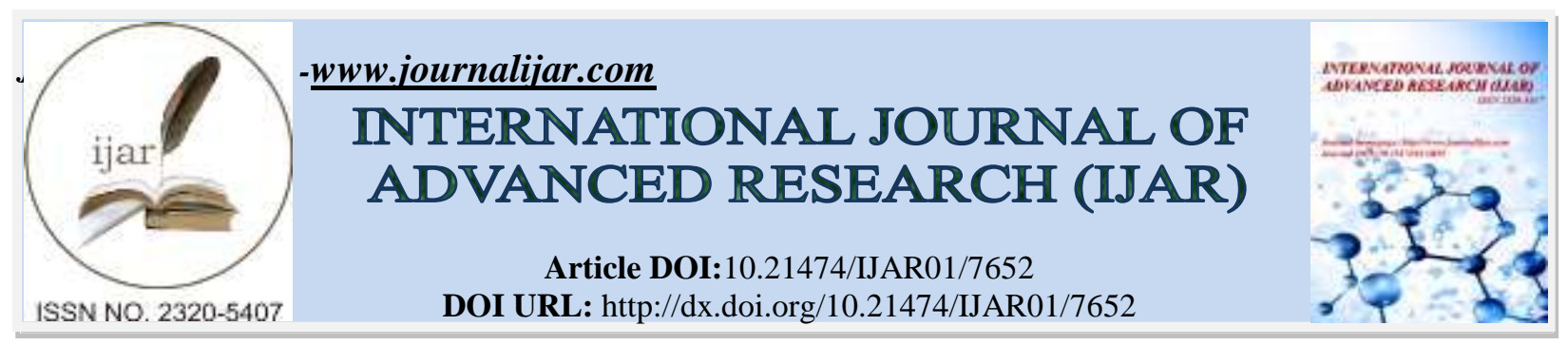

RESEARCH ARTICLE

\title{
WHEN THE TREATMENT OF A PATHOLOGY AGGRAVATES ANOTHER.TERBINAFINE AND PSORIASIS: TWO CASES REPORT.
}

\author{
Jawad El-Azhari, Youssef Zemmez, Naoufal Hjira and Mohammed Boui.
}

Service de dermatologie-vénérologie, Hôpital d'instruction Mohammed V, Rabat-Maroc.

\section{Manuscript Info}

(.........................

Manuscript History

Received: 03 July 2018

Final Accepted: 05 August 2018

Published: September 2018

Keywords:-

terbinafine, psoriasis, interleukin-23 (IL-23), latency..

\section{Abstract}

Terbinafine is an allylamine having an in vitro fungicidal effect on dermatophytes and fungistatic on Candida albicans. It is an agent widely used in dermatology but also in general medicine and other specialties. It is, however, responsible of side effects in $10.5 \%$ of patients, mainly gastrointestinal and cutaneous. These cutaneous manifestations occur in $2.7 \%$ of patients in the form of erythema, urticaria, eczema, pruritus or rarely of alopecia areata. Cases of erythema multiforme / toxic epidermal necrolysis and Stevens-Johnson syndrome have been reported. We report the cases of two patients who had psoriasis lesions after taking oral terbinafine.

Copy Right, IJAR, 2018,. All rights reserved.

\section{Introduction:-}

La terbinafine est une allylamine ayant un effet fungicide in vitro sur les dermatophytes et fungistatique sur le Candida albicans. C'est un agent largement utilisé en dermatologie mais aussi en médecine générale et dans d'autres spécialités. Il est cependant responsable d'effets secondaires chez $10,5 \%$ des patients [1], principalement gastrointestinaux et cutanés. Ces manifestations cutanées surviennent chez $2,7 \%$ des patients sous forme d'érythème, d'urticaire, d'eczéma,de prurit ou rarement de pelade. Des cas d'érythème polymorphe/nécrolyse épidermique toxique et de syndrome de Stevens-Johnson ont été signalés [2,3]. Nous rapportons les cas de deux patients présentant un psoriasis suite à la prise de terbinafine orale.

\section{Observations:-}

Observation.1: homme de 40 ans, diabétique sous régime seul depuis 2 ans, suivi pour psoriasis en plaques depuis 04 ans; et sans lésions cutanées apparente depuis 10 mois; consulte pour une éruption faite de lésions érythématosquameuses prurigineuses évoluant depuis 01 semaine, 10 jours suite à la prise de terbinafine orale à la dose de $250 \mathrm{mg} / \mathrm{jr}$ pour une onychomycose sans confirmation mycologique. Les lésions sont apparues sur le tronc puis se sont rapidement étendues jusqu' à atteindre tout le tégument, tout en continuant à prendre le traitement. Aucune autre prise médicamenteuse n'a été rapportée. L'état général était conservé, sans fièvre ni autres signes généraux. L'examen clinique trouve des lésions psoriasiformes en gouttes et en plaques confluentes par endroits, atteignant le cou, le tronc, les dos des mains, et les membres (Figure.1). Le diagnostic de psoriasis a été confirmé par l'histologie. La terbinafine a été suspendue et le patient mis sous émollient et photothérapie UVB sans nette amélioration, d' où

Corresponding Author:- Jawad El-Azhari.

Address:- Service de dermatologie-vénérologie, Hôpital d’instruction Mohammed V, Rabat-Maroc. 
le recours au methotréxate à raison de $25 \mathrm{mg} / \mathrm{semaine}$, suite auquel les lésions ont bien évoluées et ont disparu en 02 mois.

Observation.2: homme 37 ans ayant un oncle suivi pour psoriasis, sans autres antécédents notables, qui consulte pour des lésions érythémato-squameuses prurigineuses évoluant depuis 15 jours. L'examen objective des lésions d'aspect et de topographie évoquant un psoriasis (lésions érythémato-squameuses en gouttes et en plaques au niveau des zones bastion) (Figure.2). Le reste de l'examen était sans particularités. En reprenant l'interrogatoire, le patient rapporte avoir pris de la terbinafine orale à $250 \mathrm{mg} / \mathrm{jr}$ qu'un médecin lui avait prescrit pour une onychomycose à Trichophyton rubrum, 08 jours avant l'apparition des lésions. La terbinafine a été arrêtée, et le patient mis sous émollient et dermocorticoïdes forts avec une bonne évolution et blanchiment des lésions en 60 jours.

\section{Discussion:-}

Le psoriasis est une dermatose inflammatoire chronique multifactorielle. Sur un terrain génétiquement prédisposé, des facteurs environnementaux peuvent déclencher cette pathologie comme le stress, l'infection, les traumatismes... ou plus rarement des médicaments. La terbinafine peut être responsable du développement du psoriasis de novo ou de son exacerbation [4], et l'interleukine-23, suite à sa stimulation par cette dernière, pourrait jouer un rôle dans la pathogénie [5].

Tsankov et al. ont classés les médicaments inducteurs de psoriasis en trois catégories, la terbinafine appartenait au groupe de médicaments à propos desquels il existe des données considérables mais insuffisantes à l'appui de l'induction ou de l'aggravation du psoriasis [6]. La période de latence entre l'initiation du traitement par terbinafine et l'apparition des lésions cutanées est courte, de moins de 04 semaines [7,8,9]. Chez nos deux patients, la période de latence était respectivement de dix et huit jours chez le premier et le deuxième malade.

La terbinafine peut être responsable de plusieurs formes cliniques, à savoir le psoriasis en plaques (comme pour nos deux patients), le psoriasis pustuleux généralisé et le psoriasis inversé [10,11].

La distinction entre un psoriasis induit et déclenché est primordiale pour une prise en charge adéquate. Dans le psoriasis induit, le retrait de la molécule responsable conduit à une amélioration, et dans ce cas les émollients seuls sont suffisants, tandis que pour le psoriasis déclenché, on a souvent recours à la fois aux traitements topiques et systémiques [12]. Les résultats rapportés dans nos deux cas reflètent ceux de la littérature. Alors que pour le premier cas on a du avoir recours à la photothérapie et au methotréxate, nous nous sommes contentés d'un émollient et d'un dermocorticoïde chez le deuxième patient.

\section{Conclusion:-}

Nos deux cas montrent que le praticien doit être au courant des effets secondaires potentiels des médicaments qu'il prescrit chez le patient psoriasique. La terbinafine ne devrait pas être prescrite en première intention chez ces patients, et dans le cas échéant, une surveillance clinique serait nécessaire.

Conflits d'intérêts: Les auteurs ne déclarent aucun conflit d'intérêts. 


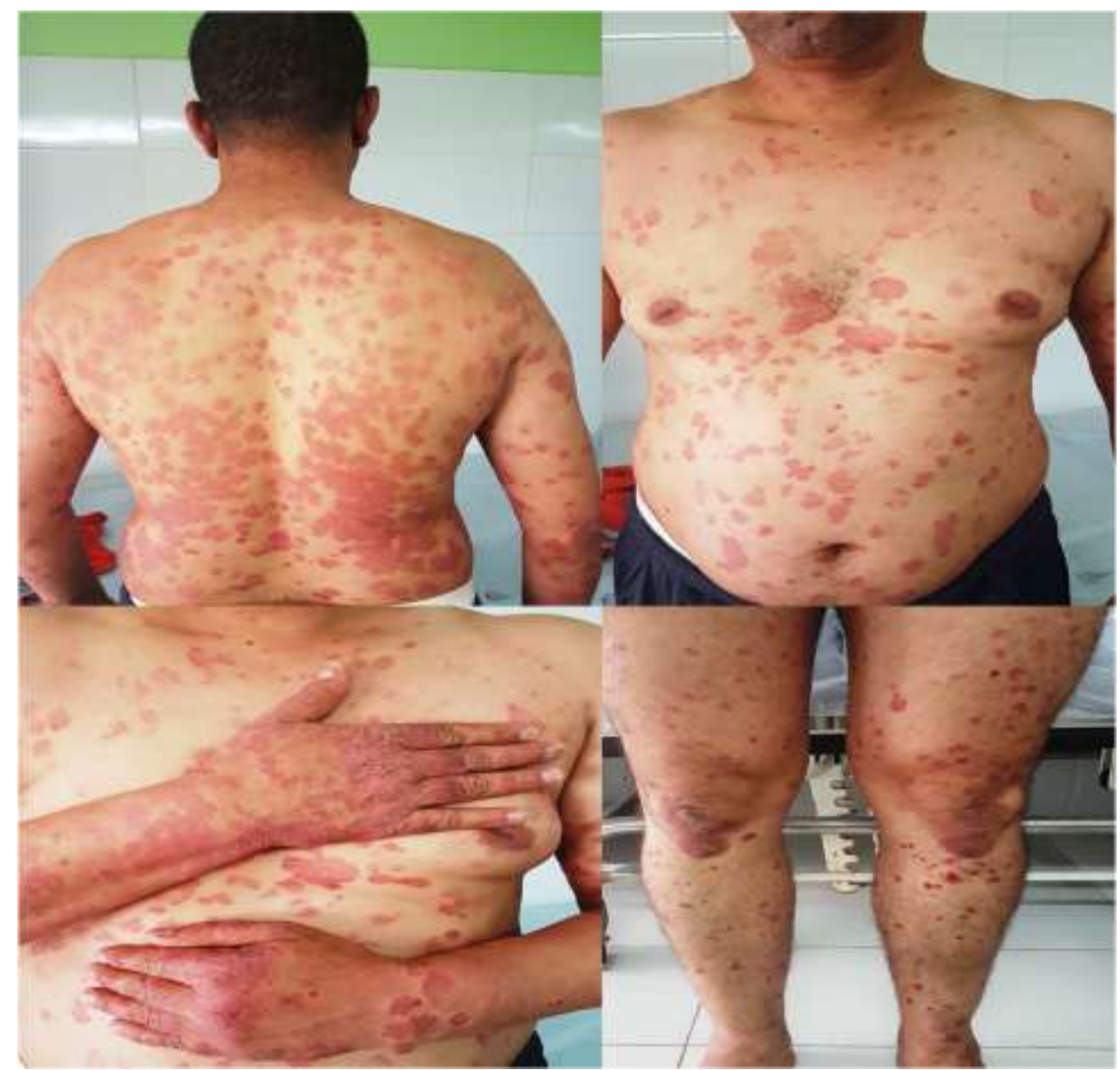

Figure.1:-lésions psoriasiformes en gouttes et en plaques confluentes par endroits, atteignant le cou, le tronc, les dos des mains, et les membres.

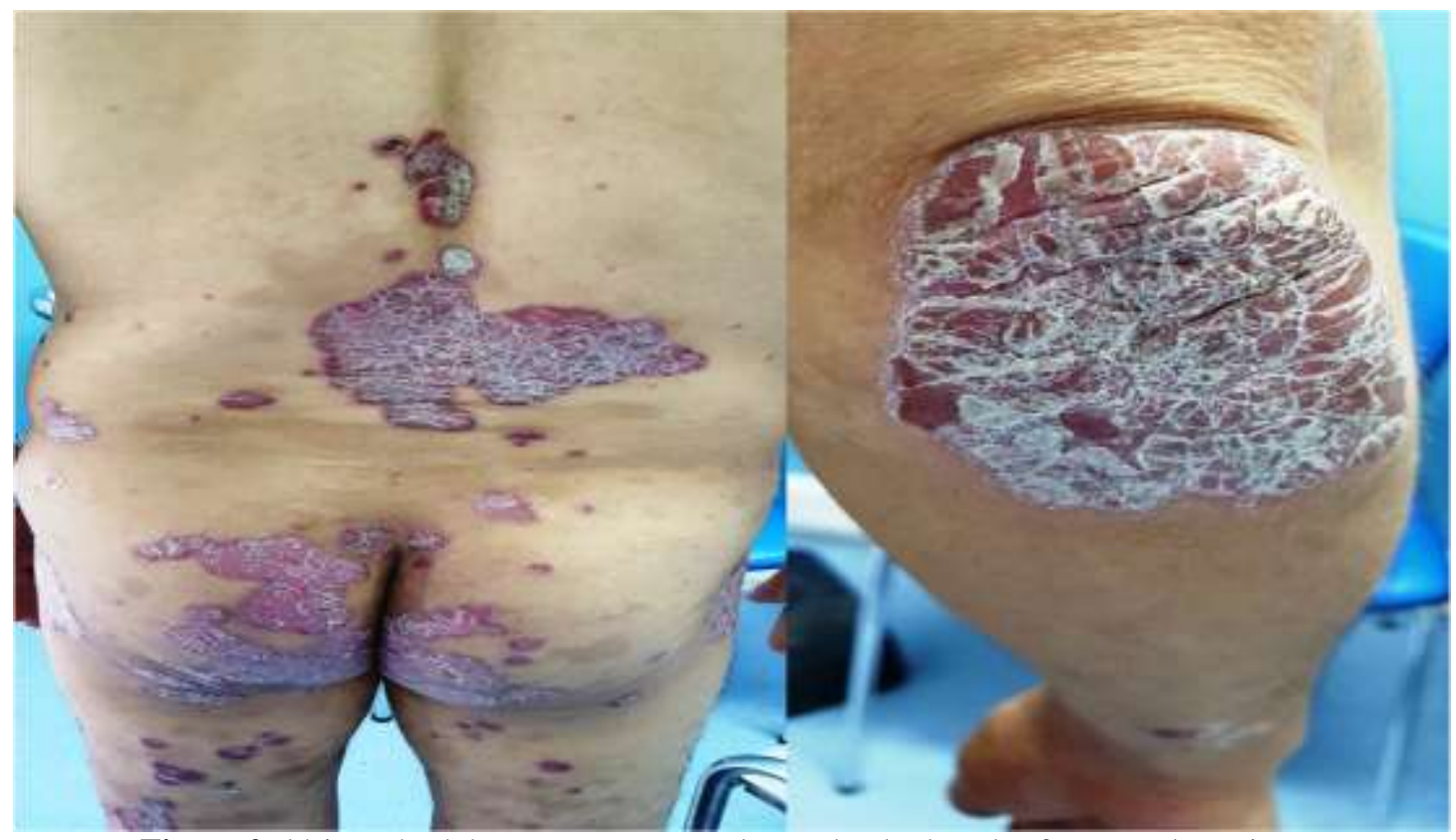

Figure.2: lésions érythémato-squameuses du coude, du dos, des fesses et des cuisses. 


\section{Référence:-}

1. Hall, M., Monka, C., Krupp, P., O’Sullivan, D., 1997. Safety of oral terbinafine: results of a postmarketing surveillance study in 25,884 patients. Arch. Dermatol. 133, 1213-1219.

2. Villars VV, Jones TC. Special features of the clinical use of oral terbinafine in the trealment of fungal diseases. Br J Dermatol 1992:126:61-9.

3. White SI, Bowen-Jones D. Toxic epidermal necrolysis induced by terbinafine in a patient on long-term antiepileptics. Br J Dermatol 1996;134:178-92.

4. Wilson, N.J., Evans, S., 1998. Severe pustular psoriasis provoked by oral terbinafine. Br. J. Dermatol. 139 , 168.

5. Oda T, Sawada Y, Yamaguchi T, et al. Psoriatic erythroderma caused by terbinafine: a possible pathogenetic role for IL-23. J Investig Allergol Clin Immunol. 2017;27(1):63-64.

6. Tsankov N, Kazandjieva J, Drenovska K. Drugs in exacerbation and provocation of psoriasis. Clin Dermatol 1998; 16: 333-51

7. Wach F, Stolz W, Rüdiger R, et al. Severe erythema annulare centrifugum-like psoriatic eruption induced by terbinafine. Arch Dermatol 1995; 131:960-1.

8. Skoven I, Thormann J. Lithium compound treatment and psoriasis. Arch Dermatol 1979;115:1185-7.

9. Eriksen JG. Christiansen JJ, Assmussen I. Pustulosis palmoplantaris caused by angiotensin-converting enzyme inhibitors. Ugeskr Laeger 1995;157:3335-6.

10. Gupta AK, Sibbald RG, Knowles SR, Lynde CW, Shear NH. Terbinafine therapy may be associated with the development of psoriasis de novo or its exacerbation: four case reports and a review of drug-induced psoriasis. $\mathbf{J}$ Am Acad Dermatol. 1997;36:858-862.

11. Kim BS, Jang HS, Jwa SW, et al. Generalized pustular psoriasis and hepatic dysfunction associated with oral terbinafine therapy. J Korean Med Sci. 2007;22:167-169.

12. Doyon JB, Liu KJ, Berman RA. Metoprolol-induced total body erythroderma. J Gen Intern Med. 2017;32(2):221-222.

13. Stanford CW, Kollipara R, Melookaran AM, Hall JC. Palmoplantar pustular psoriasis following initiation of a beta-blocker: disease control with low-dose methotrexate. Cutis. 2014;94(3):153-155. 